\title{
ON GROUPS WITH ONE DEFINING RELATION HAVING AN ABELIAN NORMAL SUBGROUP
}

A. KARRASS AND D. SOLITAR

1. Introduction. Moldavanski [6] proved that a noncyclic abelian subgroup $A$ of a group $G$ with a single defining relation is either (i) free abelian of rank 2 or (ii) locally cyclic. Even if we impose the additional restriction that $A$ be normal, the possibilities (i) and (ii) can still occur. For example, the groups

$$
G=\left\langle a, b ; a^{-1} b a=b^{k}\right\rangle, \quad k \neq 0,
$$

have abelian normal subgroups of type (i) when $k= \pm 1$ and of type (ii) otherwise. In this note we prove that the above examples are the only groups with one defining relation having noncyclic abelian normal subgroups.

2. Preliminary remarks. Let

$$
G=\langle a, b, c, \cdots ; R(a, b, c, \cdots)\rangle,
$$

$G$ noncyclic. If $R$ does not involve all the generators, then $G$ is a nontrivial free product of a free group and a group with one defining relator, and therefore $G$ has no nontrivial abelian normal subgroup. Hence we may assume that $G$ is finitely generated and that $R(a, b, c, \cdots)$ is cyclically reduced and involves all the generators. We may also assume that $R(a, b, c, \cdots)$ has zero exponent sum on some generator, say $a$ (denoted $\sigma_{a}(R)=0$ ).

As is well known (see Magnus [4] or pp. 254-257 in [5]), a presentation of the normal subgroup $H$ of $G$ generated by $b, c, \cdots$ is given as follows: Its generators are

$$
b_{k}=a^{k} b a^{-k}, \quad c_{k}=a^{k} c a^{-k}, \cdots, \quad k=0, \pm 1, \pm 2, \cdots .
$$

and its defining relators are

$R_{k}=R_{0}\left(b_{\lambda+k}, \cdots, b_{\mu+k}, c_{\rho+k}, \cdots, c_{\sigma+k}, \cdots\right), \quad k=0, \pm 1, \pm 2, \cdots$, where $R_{0}\left(b_{\lambda}, \cdots, b_{\mu}, c_{\rho}, \cdots, c_{\sigma}, \cdots\right)$ is the word obtained by rewriting $R(a, b, c, \cdots)$ in terms of the $b_{k}, c_{k}, \cdots$. Since $R(a, b, c, \cdots)$ is cyclically reduced, so is $R_{k}$.

Using this presentation for $H$, it can be shown that $H$ is the union of the ascending chain of groups (amalgamated products)

Received by the editors February 4, 1969. 


$$
K_{k}=H_{-k} *_{J_{-k}} H_{-k+1} *_{J_{-k+1}} \cdots *_{J_{k-1}} H_{k}
$$

where

$$
\begin{aligned}
H_{0} & =\left\langle b_{\lambda}, \cdots, b_{\mu}, c_{\rho}, \cdots, c_{\sigma}, \cdots ; R_{0}\right\rangle, \\
H_{i} & =\left\langle b_{\lambda+i}, \cdots, b_{\mu+i}, c_{\rho+i}, \cdots, c_{\sigma+i}, \cdots ; R_{i}\right\rangle,
\end{aligned}
$$

and $J_{i}$ is the free group

$$
J_{i}=\left\langle b_{\lambda+i+1}, \cdots, b_{\mu+i}, c_{\rho+i+1}, \cdots, c_{\sigma+i}, \cdots\right\rangle .
$$

We define

$$
H_{i, j}=H_{i} *_{J i} \cdots *_{J j-1} H_{j}, \quad i \leqq j .
$$

(Clearly $K_{m}=H_{-m, m}$.) Then

$$
H_{i, k}=H_{i, j} *_{J j} H_{j+1, k}, \quad i \leqq j<j+1 \leqq k .
$$

From this it follows easily that

$$
H_{i, j} \cap H_{t, u}<J_{j} \cap J_{t-1}
$$

where $i \leqq j<t \leqq u$.

Lemma 1 is implicit in Magnus [4] and is also proved in Moldavanski [6].

Lemma 1. Let

$$
L=\langle x, y, z, \cdots ; S(x, y, z, \cdots)\rangle
$$

where $S(x, y, z, \cdots)$ is cyclically reduced. If $x=U(y, z, \cdots)$ in $L$ then the word $S(x, y, z, \cdots)$ is (up to cyclic permutation) the word $x^{-1} U(y, z, \cdots)$ or its inverse.

We may extend Theorem 1 of Murasugi [7] to the following by using a result of Epstein [2]:

LEMMA 2. Let

$$
G=\langle a, b, c, \cdots ; R(a, b, c, \cdots)\rangle
$$

with at least three generators. Then $G$ contains no nontrivial abelian normal subgroup.

Proof. We may assume $R$ is cyclically reduced, $\sigma_{a}(R)=0$, and $R$ involves all the generators of the presentation. Suppose $N \neq 1$ is an abelian normal subgroup of $G$. Let $H$ be the normal subgroup of $G$ generated by $b, c, \cdots$.

If $N \cap H=1$, then $N$ is cyclic and is generated by some element $a^{k} h$ where $k>0$ and $h$ is in $H$. For any $g$ in $G, g^{-1}\left(a^{k} h\right) g=\left(a^{k} h\right)^{t}$ and 
so $k=k t$, i.e., $t=1$. Thus $a^{k} h$ is in the center of $G$ and by Murasugi [7], $G$ has two generators.

Hence $N \cap H \neq 1$, i.e., $H$ contains a nontrivial abelian normal subgroup. Now $H$ can be written in the form

$$
H=L_{-1} *_{J_{-1}} H_{0} *_{J_{0}} L_{1}
$$

it is easy to show that $L_{-1} \neq J_{-1} \neq H_{0} \neq J_{0} \neq L_{1}$. But an abelian normal subgroup of such an amalgamated product $H$ is contained in $J_{0}$ (by Epstein [2], since a subgroup of a factor cannot be of finite index in a nontrivial amalgamated product). Thus the free group $J_{0}$ contains a nontrivial abelian normal subgroup and so $J_{0}$ must be cyclic, i.e., $J_{0}=\operatorname{gp}\left(b_{\lambda+1}\right)$. Now in $H_{0}$ the elements $b_{\lambda+1}$ and $c_{\rho}$ freely generate $\operatorname{gp}\left(b_{\lambda+1}, c_{\rho}\right)$. But this last subgroup contains $N \cap H$; this is impossible, and so $G$ cannot contain a nontrivial abelian normal subgroup.

\section{Noncyclic abelian normal subgroups.}

Theorem 1. Let $G=\langle a, b ; R(a, b)\rangle$ where $\sigma_{a}(R)=0$. Then $G$ contains an infinitely generated abelian normal subgroup iff

$$
R(a, b,)=\left(a^{\epsilon} b a^{-\epsilon} b^{-k}\right)^{\eta}, \quad \eta= \pm 1
$$

(up to cyclic permutation) where $|\epsilon|=1$ and $|k|>1$.

Proof. Clearly (2) implies that $b_{\epsilon}=b_{0}^{\boldsymbol{k}}$, so that $H$ is a union of a proper ascending chain of infinite cyclic groups; $H$ is therefore an infinitely generated locally cyclic abelian normal subgroup.

Conversely, suppose $G$ contains an infinitely generated abelian normal subgroup $N$. Then $N \cap H$ is an infinitely generated abelian normal subgroup of $H$. If $H_{0} \neq J_{0} \neq H_{1}$, then as in the proof of Lemma 2, $N \cap H$ is contained in $J_{0}$ and $N \cap H$ is therefore cyclic. Hence $H_{0}=J_{0}$ or $H_{1}=J_{0}$; say $H_{0}=J_{0}<H_{1}$. This implies $H=\bigcup_{i=0}^{\infty} J_{i}$, and so $N \cap H=\cup_{i=0}^{\infty}\left(N \cap J_{i}\right)$, so that $N \cap J_{i} \neq 1$ for $i \geqq t$, say. Therefore the free group $J_{i}$ contains a nontrivial abelian normal subgroup, and so $J_{i}$ is cyclic and $J_{i} \neq J_{i+1}$. Thus $J_{0}=\mathrm{gp}\left(b_{\lambda+1}\right)$ and $b_{\lambda}=b_{\lambda+1}^{k}$ where $|k|>1$. Then by Lemma $1, R_{0}^{\epsilon}=b_{\lambda} b_{\lambda+1}^{-k}$ (up to cyclic permutation), i.e., $R(a, b)$ is cyclically equal to $a^{-1} b a b^{-k}$ or its inverse. Similarly if $H_{1}=J_{0}$, we obtain $R(a, b)$ is (up to inverse) cyclically equal to $a b a^{-1} b^{-k}$.

We need now concern ourselves only with nontrivial finitely generated abelian normal subgroups.

Theorem 2. Let $G=\langle a, b ; R(a, b)\rangle$ where $\sigma_{a}(R)=0$. Let $N$ be $a$ finitely generated abelian normal subgroup of $G$. Then the rank of $N$ is 
$\leqq 2$, with equality possible only if $R(a, b)=a b a^{-1} b^{-\epsilon}$ or its inverse (up to cyclic permutation) where $|\epsilon|=1$.

Proof. By [6], the rank of $N \leqq 2$. Suppose $N$ has rank two. If $N \cap H=1$, then $N$ is infinite cyclic. Therefore $N \cap H \neq 1$. Since $N \cap H$ is finitely generated, $N \cap H<K_{m}$ for some $m$. But then

$$
N \cap H=a^{2 m+1}(N \cap H) a^{-2 m-1}<L
$$

where $L=H_{m+1,3 m+1}$. Hence, by (1), $N \cap H<J_{m}$ and so $N \cap H<J_{0}$. Thus $J_{0}$ is infinite cyclic, and so too is $N \cap H$. Moreover, if $N \cap H$ $=\operatorname{gp}\left(b_{\lambda+1}^{r}\right)=\operatorname{gp}\left(b^{r}\right)$, then since $N \cap H$ is normal in $G, a b^{r} a^{-1}=b^{\text {er }}$ where $|\epsilon|=1$, and so

$$
b_{\delta}^{r}=b^{r \mathrm{e}^{\delta}} .
$$

Thus $N \cap H$ is in the center of $H$.

Since $N$ has rank 2, $N=\operatorname{gp}\left(a^{k} h, b^{r}\right)$ where $k>0$ and $h$ is in $H$. We first show that $h=b_{r}^{s}$. For, we may choose $q$ so large that $h, b$ are both in $K_{q}$. Now

$$
a\left(a^{k} h\right) a^{-1}=a^{k}\left(a h a^{-1}\right)=\left(a^{k} h\right)^{\alpha}\left(b^{r}\right)^{\beta} .
$$

Therefore $k=k \alpha$, i.e., $\alpha=1$. Hence $a h a^{-1}=h b^{r \beta}$, and it is easy to see that

$$
a^{n} h a^{-n}=h b^{r \beta_{n}} .
$$

The right-hand side of (4) is in $K_{q}$ while the left-hand side of (4) as well as $b^{r}$ is in $H_{-q+n, q+n}$. Letting $n=2 q+1$, we see that $h$ is in $J_{q}$, and so $h=b_{\tau}^{s}$ (where $\left.\tau=\lambda+q+1\right)$. Thus

$$
N=\operatorname{gp}\left(a^{k} b_{\tau}^{8}, b^{\tau}\right)=\operatorname{gp}\left(a^{k} b^{s}, b_{-\tau}^{r}\right)=\operatorname{gp}\left(a^{k} b^{s}, b^{r}\right) .
$$

Moreover,

$$
b a^{k} b^{s} b^{-1}=a^{k} b_{-k} b^{s-1}=\left(a^{k} b^{s}\right)^{\rho}\left(b^{r}\right)^{\sigma} ;
$$

and so $k=k \rho$, i.e., $\rho=1$ so that $b_{-k}=b^{r \sigma+1}$. Now by (3),

$$
b_{-k}^{r}=b^{r e^{-k}}=b^{r(r \sigma+1)} \text {. }
$$

Therefore $r \sigma+1=\epsilon^{-k}$, and so $b_{-k}=b^{-k}$. More generally, $b_{t k}=b^{t k}$. Therefore $b$ is in $H_{t k}$ for all integers $t$, so that by (1), $b$ is in $\bigcap_{-\infty}^{\infty} J_{i}$. In particular, $b$ is in $J_{-\lambda}=\mathrm{gp}\left(b_{1}\right)$ and $b_{1}$ is in $J_{-\lambda-1}=\mathrm{gp}(b)$. Hence, $b_{1}=b^{\epsilon}, \epsilon= \pm 1$. Consequently, by Lemma 1 ,

$$
R_{-\lambda}\left(b_{0}, b_{1}\right)=\left(b_{1} b_{0}^{-c}\right)^{\eta}, \quad \eta= \pm 1
$$


(up to cyclic permutation) which implies $R(a, b)$ is cyclically equal to $a b a^{-1} b^{-\epsilon}$ or its inverse.

If $R(a, b)$ is of this form, then clearly $\mathrm{gp}\left(a^{2}, b\right)$ is an abelian normal subgroup of $G$ of rank 2 .

\section{Additional remarks.}

THEOREM 3. Let

$$
G=\left\langle a, b, c, \cdots ; R^{q}(a, b, c, \cdots)\right\rangle
$$

where $q>1$. Then $G$ has no nontrivial normal abelian subgroup unless $G$ is cyclic.

Proof. By Lemma 2, we may assume $G=\left\langle a, b ; R^{a}(a, b)\right\rangle$ where $\sigma_{a}(R)=0$, and $R$ is cyclically reduced and involves both $a$ and $b$. Since $a^{e} b a^{-\varepsilon} b^{-k}$ is not a true power, Theorem 1 implies that $G$ has no infinitely generated abelian normal subgroup.

Suppose that $N$ is a nontrivial normal abelian subgroup of $G$.

If $N \cap H \neq 1$, then the proof of Theorem 2 shows that $N \cap H$ $=\mathrm{gp}\left(b^{r}\right)$ is in the center of $H$ and therefore in the center of $H_{-\lambda}$. But

$$
H_{-\lambda}=\left\langle b_{0}, b_{1} ; R_{-\lambda}^{q}\left(b_{0}, b_{1}\right)\right\rangle,
$$

a group with trivial center (see Theorem 4, Corollary in [3]).

Hence $N \cap H=1$ and $N$ is in the center of $G$, which is again impossible.

Finally, using the preceding results and an algorithm of Baumslag and Taylor [1], we show how to determine all the abelian normal subgroups of a group with a single defining relation.

Let $G=\langle a, b ; R(a, b)\rangle$ where $\sigma_{a}(R)=0$.

Theorem 1 gives a nasc for $G$ to have an infinitely generated abelian normal subgroup. Moreover, if $R(a, b)$ is as in (2), then $H$ is an infinitely generated abelian normal subgroup; if $N$ is any nontrivial abelian normal subgroup, then $N$ is infinitely generated and in fact $N=H^{d}$, the $d$ th powers of the elements of $H$.

Theorem 2 gives a nasc for $G$ to have an abelian normal subgroup of rank 2. If $R(a, b)=a b a^{-1} b$ then the abelian normal subgroups of $G$ are the following:

$$
\mathrm{gp}\left(a^{2 t}\right), \quad \mathrm{gp}\left(b^{s}\right), \quad \operatorname{gp}\left(a^{2 t}, b^{s}\right), \quad \operatorname{gp}\left(a^{2 t} b^{s}, b^{2 s}\right)
$$

where $s, t$ are any integers.

We need then only concern ourselves with the groups all of whose abelian normal subgroups are cyclic. Using the algorithm of Baumslag 
and Taylor [1], we can determine the center $Z(G)$ of $G$ and therefore all subgroups of $Z(G)$. In particular, if $N \cap H=1$, then $N<Z(G)$. Assume $N<H$; therefore as in the proof of Theorem 2, $N=\operatorname{gp}\left(b^{r}\right)$ and $a b^{r} a^{-1}=b^{e r}, \epsilon= \pm 1$. Assume $r$ minimal. Since $b_{\lambda}^{r}$ must be in the center of

$$
H_{0}=\left\langle b_{\lambda}, b_{\lambda+1} ; R_{0}\left(b_{\lambda}, b_{\lambda+1}\right)\right\rangle,
$$

we may use the methods in [1] to bound $r$, and then use the solution of the word problem in $G$ to determine whether $a b^{r} a^{-1}=b^{e r}$.

\section{REFERENCES}

1. G. Baumslag and T. Taylor, The centre of groups with one defining relator, Math. Ann. 175 (1968), 315-319.

2. D. B. A. Esptein, A result on free products with amalgamation, J. London Math. Soc. 37 (1962), 130-132.

3. A. Karrass, W. Magnus and D. Solitar, Elements of finite order in groups with a single defining relation, Comm. Pure Appl. Math. 13 (1960), 57-66.

4. W. Magnus, Über diskontinuierliche Gruppen mit einer definierenden Relation (der Freiheitssatz), J. Reine Angew. Math. 163 (1930), 141-165.

5. W. Magnus, A. Karrass and D. Solitar, Combinatorial group theory, Interscience, New York, 1966.

6. D. I. Moldavanski, Certain subgroups of groups with a single defining relation, Sibirsk. Mat. Z. 8 (1967), 1370-1384.

7. K. Murasugi, The center of a group with a single defining relation, Math. Ann. 155 (1964), 246-251.

York UnIversity, ToRonto 